\title{
Cet - Centro de Estudos das Toxicomanias Dr. Claude Olievenstein da UFPR ${ }^{1}$
}

\section{UFPR Center of Studies on Drug Addiction Doctor Claude Olievenstein}

\section{CET - Centro de Estudios de las Toxicomanías Doctor Claude Olievenstein de la UFPR}

\author{
Victor Eduardo Silva Bento² \\ Marcos Alan Viana ${ }^{3}$
}

\begin{abstract}
RESUMO
Trata-se de artigo de relato de experiência de extensão universitária na área da saúde. Relatou-se aqui a experiência profissional de criação e direção do CET - Centro de Estudos das Toxicomanias Dr. Claude Olievenstein: Pesquisa, Ensino, Tratamento e Prevenção. Foram narrados sua pré-história e seu nascimento em 19 de outubro de 1999 no Setor de Ciências Humanas, Letras e Artes da Universidade Federal do Paraná. Foi criado por professores e profissionais da UFPR, sob a liderança do Prof. Dr. Victor Eduardo Silva Bento do Departamento de Psicologia, primeiro diretor deste Centro desde sua criação até maio de 2005. Desde seu nascimento, o CET possuía um Acordo de Cooperação com o Centre Médical Marmottan/Paris, este nesta ocasião sob a direção de Claude Olievenstein. Foram também descritas as atividades de Pesquisa, Ensino e Extensão Clínica do CET, segundo a orientação teórica principal da psicanálise, em debate inter e multidisciplinar. Finalmente, avaliaram-se as ações desenvolvidas no CET, destacando-se os prêmios e o financiamento recebidos.

Palavras-chave: Centro de Estudos das Toxicomanias; Universidade Federal do Paraná; Claude Olievenstein.
\end{abstract}

\begin{abstract}
This is a university extension study about reported experience on the health area. This article describes the professional experience of launching and administrating the "Center of Studies on Drug Addiction Doctor Claude Olievenstein: Research, Teaching, Treatment and Prevention". This paper also refers to the prehistory and the launching of that Center which was on October 19th 1999 . The Center was part of the School of Humanities of the Federal University of Paraná - UFPR. It was created by a group of UFPR professors and professionals led by Victor Eduardo Silva Bento, professor of the Psychology Department. Professor Bento was the first Center director up to may, 2005. Since its beginning, the Center has had a Cooperative
\end{abstract}

\footnotetext{
1 O conteúdo deste trabalho foi extraído do Banner do CET, não publicado, intitulado "Sobre o CET da UFPR", de autoria dos mesmos autores do presente trabalho, confeccionado em agosto de 2004. Alguns dados foram acrescentados em 2009 para compor o conteúdo final do presente trabalho.

2 Doutor em Psicopatologia Fundamental e Psicanálise pela Universidade Paris 7; Ex-Professor do Departamento de Psicologia da UFPR (02/1984 - 05/2005); Ex-Diretor do CET - Centro de Estudos das Toxicomanias Dr. Claude Olievenstein da UFPR (10/1999 - 05/2005); Professor do Departamento de Psicometria do Instituto de Psicologia da UFRJ (desde 09/2005). Endereço: Rua Domingos Ferreira 180 / cob. 02 - Copacabana / Rio de Janeiro / CEP 22050-010. Fone / Fax: (21) 25472167. E-mails: vsilvabento@yahoo.com.br; victorbento@oi.com.br.

3 Bolsista de Iniciação Científica do CET da UFPR (2002), Especialista em Psicanálise das Toxicomanias pelo CET da UFPR e Psicólogo da Associação Projeto Não Violência Brasil. Endereço: Rua Paulino Siqueira Côrtes 282 / apto.2 - Silveira da Motta / São José dos Pinhais / Paraná / CEP 83030-730. Fone: (41) 33851235. E-mail: alanpsico@yahoo.com.br.
} 
Agreement with the Centre Médical Marmottan/Paris, at the time directed by Professor Claude Olievenstein. The CET activities of Research, Teaching and Clinical Extension, under the main theoretical orientation of psychoanalysis, debating with other theoretical orientations, were described. Finally, the Center prizes and grants were mentioned.

Keywords: Center of Studies on Drug Addiction; Federal University of Paraná; Claude Olievenstein.

\section{RESUMEN}

Se trata de relato de la experiencia de extensión universitaria en el dominio da la salud. Se relató acá la experiencia profesional de creación y dirección del "CET - Centro de Estudios de las Toxicomanías Doctor Claude Olievenstein: Pesquisa, Enseñanza, Tratamiento y Prevención”. Se narró suya prehistoria y su nacimiento en 19 de octubre de 1999 en el Sector de Ciencias Humanas, Letras y Artes de la UFPR - Universidad Federal del Paraná. Fue criado por profesores y profesionales de la UFPR, bajo la dirección del profesor Victor Eduardo Silva Bento del Departamento de Psicología, primero director de este Centro desde suya creación hasta mayo de 2005. Desde su nacimiento, el CET poseía un Acuerdo de Cooperación con el Centre Médical Marmottan/Paris, este en esa ocasión bajo la dirección de Claude Olievenstein. Fueron también describidlas las acciones de Pesquisa, Enseñanza y Extensión Clínica del CET, siguiendo la orientación teórica principal de la psicoanálisis, en debate Inter. y multidisciplinar. Finalmente, valuaron-se las acciones realizadas en el CET, destacando-se los premios y lo financiamiento recibidos.

Palabras-clave: Centro de Estudios de las Toxicomanías; Universidad Federal del Paraná; Claude Olievenstein.

\section{Introdução}

O objetivo deste artigo é relatar a experiência profissional do Prof. Dr. Victor Eduardo Silva Bento de criação e direção do CET - Centro de Estudos das Toxicomanias Dr. Claude Olievenstein: Pesquisa, Ensino, Tratamento e Prevenção - da UFPR - Universidade Federal do Paraná, no período de 19/10/99 a 02/05/05. Tratava-se de um Centro de Pesquisa, Ensino e Extensão, de orientação psicanalítica, em debate inter e multidisciplinar, criado por um grupo de servidores da UFPR, sob a liderança de Bento, com um Acordo de Cooperação com o Centre Médical Marmottan/Paris, este último centro, nesta ocasião da criação do CET, sob a direção do Prof. Dr. Claude Olievenstein.

Justifica-se a importância deste artigo pelos seguintes motivos: 1 - aborda as toxicomanias, uma problemática que está na ordem do dia; 2 - é original, pois relatará uma abordagem das toxicomanias valorizando o enfoque teórico da psicanálise, em debate inter e multidisciplinar, no contexto da universidade, o que é pouco explorado na literatura. Em geral, é mais frequente encontrar trabalhos sobre as toxicomanias segundo a orientação médica ou comportamental; também é questionável pensar na possibilidade de uma psicanálise das toxicomanias, e ainda em debate inter e multidisciplinar; finalmente, é igualmente polêmico falar em transmissão do saber psicanalítico na universidade; 3 - permitirá a divulgação do trabalho que foi realizado no CET da UFPR, no período de outubro de 1999 a maio de 2005, o que se supõe que facilitará o intercâmbio com outros pesquisadores $e$ profissionais interessados na problemática das toxicomanias abordada segundo a orientação principal da psicanálise, em debate inter $e$ multidisciplinar, no contexto acadêmico-universitário; 4 - permitirá, com sua publicação, um registro histórico de uma experiência acadêmicoinstitucional bem sucedida no campo da psicanálise das toxicomanias na universidade.

O que foi o CET da UFPR no período de outubro de 1999 a maio de 2005?

Como o seu subtítulo indica, o CET, no período de 19 de outubro de 1999 a 2 de maio de 2005, foi um Centro de Pesquisa, Ensino, 
Tratamento e Prevenção das Toxicomanias. O Art. 3. ${ }^{\circ}$ do Regimento Interno do CET previa:

O centro tem por finalidade realizar um trabalho técnico de cunho científico no trato das toxicomanias, sob uma orientação psicanalítica aberta ao enfoque multidisciplinar e ao debate com outras orientações teóricas, valorizando o tripé fundamental que caracteriza a ideologia e a realidade das Universidades em geral: ensino, pesquisa e extensão.

\section{A Antena Marmottan no Brasil}

A influência pré-histórica que justificou a criação e a estrutura do CET no período de outubro de 1999 a maio de 2005 foi a existência, em outras capitais brasileiras, de centros de estudos das toxicomanias com Acordo de Cooperação com o Centre Médical Marmottan, fato que serviu de inspiração para chamar o título desse subitem de A Antena Marmottan no Brasil.

Apesar da existência de diversas iniciativas promovidas por diversos hospitais paranaenses, no sentido de tratar das toxicomanias, observava-se que este empreendimento se realizava, em geral, no contexto global do tratamento de outras psicopatologias. Eram poucas as ações que valorizavam o investimento específico e exclusivo na clínica das toxicomanias em âmbito hospitalar. Também não existiam no Paraná Centros de Referência, no contexto das Universidades, que se ocupassem apenas desta problemática das toxicomanias, dando-lhe uma atenção particular simultaneamente no campo da Pesquisa, do Ensino, do Tratamento e da Prevenção. Muito menos ainda existia no Paraná um serviço dedicado exclusivamente a esta problemática sob o enfoque específico e principal da psicanálise. Finalmente, inexistiam no mesmo Estado, iniciativas nesse campo da clínica das toxicomanias realizadas por instituições com Acordo de Cooperação com o Centre Médical Marmottan. Tal carência de exclusividade e especificidade só podia dificultar o aprofundamento e o enfrentamento da mesma problemática.

Caberia agora comparar o Paraná com outros estados brasileiros, para assim demonstrar as tendências atuais brasileiras na abordagem das toxicomanias e as carências desse Estado em relação às mesmas tendências. O Estado de Minas Gerais, por exemplo, criou em 1983 o CMT - Centro Mineiro de Toxicomania - Tratamento, Prevenção, Ensino e Pesquisa (GROSSI, 199?, p. 43), ligado à Fundação Hospitalar do Estado de Minas Gerais (FHEMIG)/Secretaria Estadual da Saúde. Sobre a importância da criação do CMT na história do tratamento hospitalar brasileiro das toxicomanias, estas vistas como problema de saúde pública, Grossi escreve:

\begin{abstract}
A singularidade do CMT se relaciona tanto com a história de sua criação, em agosto de 1983, quanto com sua prática, sobretudo no campo clínico, em que a psicanálise é a ferramenta que baliza a direção do tratamento $e$ o funcionamento da instituição. Fruto do movimento da Reforma Psiquiátrica e do Movimento Psicanalítico Mineiro, foi a primeira instituição pública fundada no Brasil especializada no tratamento de toxicomanias. Sua fundação representa um deslocamento importante no enfoque do poder público a respeito dos usuários: a toxicomania é um caso não de polícia, mas sim de saúde pública. (GROSSI, 2001, p.165)
\end{abstract}

Como relata Grossi (199?, p.18), com a criação do CMT "um passo fundamental foi dado: buscou-se pensar a toxicomania dentro da singularidade de cada um e o saber psicanalítico como teoria e ferramenta clínica". E para apoiar tal iniciativa, o primeiro nome citado pelo mesmo autor foi o de Olievenstein. Ele escreve: "Em setembro de 1984 realizou-se o primeiro Seminário sobre Toxicomania em nosso 
Estado contando com a presença do Dr. Claude Olievenstein, uma das maiores autoridades nessa área" (GROSSI, 199?, p.18). Sobre a realidade deste Centro, aproximadamente 10 anos depois desse seminário, isto é, em 1994, acrescenta o mesmo autor:

Realidade atual - O Centro Mineiro de toxicomania, CMT, é uma das 21 unidades da [...] FHEMIG, único Centro Público de Referência em nosso Estado, integrando uma rede de seis (6) outros Centros de Referência nos Estados de Pernambuco, Bahia, Rio de Janeiro, São Paulo, Rio Grande do Sul e Distrito Federal. Os diretores dos Centros integram o Comitê Assessor para Álcool e Drogas do Ministério da Saúde e assessoram o Conselho Federal de Entorpecentes (CONFEN) do Ministério da Justiça, órgão autorizado para credenciar os Centros de Referência (GROSSI, 199?, p.18).

Outro exemplo é o Estado do Rio de Janeiro que, três anos após a criação do CMT, isto é, em 1986, criou o Núcleo de Estudos e Pesquisas em Atenção ao Uso de Drogas (Nepad), vinculado à Universidade de Estado do Rio de Janeiro e credenciado pelo Conselho Federal de Entorpecentes. Era um Centro de Referência Regional para Prevenção e Tratamento no campo das toxicomanias. Esse Núcleo era também credenciado pelo Ministério da Saúde como Centro de Referência Nacional para Drogas e DST/AIDS. E aqui se observava a mesma tendência de contar com o apoio técnico internacional, em particular do governo francês, apoio este que perdurou no tempo. A esse respeito vale lembrar que, de 30/10/96 a 01/ 11/96, para comemorar seu décimo aniversário, o Nepad realizou o II Seminário Internacional sobre as Toxicomanias, e a participação especial neste seminário não foi outra que a do mesmo professor Claude Olievenstein.
Descendo na direção do sul, caberia ainda lembrar a experiência do Estado de São Paulo, que mais uma vez confirmou a mesma tendência acima referida. Como se encontra referido desde a contracapa do livro de Silveira Filho e Gorgulho:

[...] o PROAD - Programa de Orientação e Assistência a Dependentes, serviço ligado ao Departamento de Psiquiatria e Psicologia Médica da Escola Paulista de Medicina (Universidade Federal de São Paulo), foi criado em 1987 visando desenvolver atividades de assistência, ensino, pesquisa e prevenção na área de farmacodependências. Em 1988, o PROAD foi escolhido pelo CONFEN - Conselho Federal de Entorpecentes - como um dos cinco Centros Brasileiros de Referência na área de farmacodependências (SILVEIRA FILHO; GORGULHO, 1996, contracapa).

O Proad vem igualmente mantendo convênios de cooperação técnico-científica com algumas das mais renomadas instituições internacionais, entre as quais se destacam: 1 - Com o Centre Médical Marmottan/Paris, cuja direção era realizada pelo mesmo Professor supracitado, o Dr. Claude Olievenstein; 2 - com o Ministère des Affaires Etrangères (Paris - França); 3 - com o SOS Drogue International (Paris França). Quanto à orientação teórica do PROAD, pode-se destacar, de um lado, a existência de uma abordagem multidisciplinar, e, de outro, o fato de seu fundador e coordenador, o Dr. Dartiu Xavier da Silveira Filho, ser Membro da International Association Analytical Psychology, e definir sua abordagem como "psicodinâmica" (SILVEIRA FILHO, 1995), mas tecendo também considerações psicanalíticas.

Em resumo, a partir desta breve caracterização do CMT, do Nepad e do Proad, vê-se estampar o perfil de uma tendência atual no Brasil: a de se criar Centros de Referência na 
área das toxicomanias, reconhecidos enquanto tais pelo Confen, e contando com o apoio técnico-científico de instituições internacionais, em particular do governo francês, representado principalmente por Olievenstein. Vê-se também que tais Centros valorizam quatro grandes áreas de intervenção: Pesquisa, Ensino, Tratamento e Prevenção. Finalmente, vê-se ainda que, por um lado, encontra-se o destaque da abordagem multidisciplinar, enquanto que, por outro, parece existir certa simpatia pelo enfoque teórico da psicanálise para fundamentar e aprofundar a problemática das toxicomanias.

Portanto, pode-se destacar nesse item a importância do Paraná acompanhar uma política já implementada em outros estados brasileiros, criando Centros na área das toxicomanias ligados a Centros de Referência especializados na problemática em questão, sob a ótica principal da psicanálise, e com o apoio técnico e científico internacional, em particular do governo francês, representado por Olievenstein, uma das maiores autoridades mundiais em toxicomania, falecido em 2008, ex-diretor do Centre Médical Marmottan, um Centro de Referência especializado na desintoxicação de toxicômanos. O CET foi, então, criado em 19/10/99 na tentativa de acompanhar esta tendência aqui destacada.

2. A vinda do Prof. Dr. Claude Olievenstein a Curitiba

Nos dias 4 e 5 de novembro de 1996, Olievenstein veio pela primeira vez a Curitiba para ministrar o Curso de Extensão intitulado Drogas, violência e exclusão na infância e na adolescência, promovido pelo Departamento de Psicologia da UFPR, sob a coordenação do Prof. Bento. Olievenstein pretendia estimular a criação de um primeiro centro de estudos das toxicomanias no Paraná com um Acordo de Cooperação com o Centre Médical Marmottan, permitindo que o Estado acompanhasse esta tendência brasileira de criar Centros dessa natureza, tal como ocorria em outros estados brasileiros.
Esse evento de extensão, de novembro de 1996, foi de fato, um acontecimento memorável. Além de Olievenstein, este evento contou também com a presença de outras eminentes autoridades, como, por exemplo, o então Reitor da UFPR, Prof. Dr. José Henrique de Faria; a primeira dama do Estado do Paraná, Sra. Fani Lerner; outro ex-Reitor da UFPR, o Prof. Dr. Carlos Roberto Antunes dos Santos, então Diretor do Setor de Ciências Humanas, Letras e Artes da UFPR, etc.

No entanto, nenhum centro de estudos das toxicomanias foi criado após esta primeira visita de Olievenstein a Curitiba, o que o fez retornar nos dias 5 e 6 de novembro de 1998. Era, então, sua segunda visita a esta capital, para assim, insistir na importância de criação de um centro com tal especificidade, também nesta cidade. Olievenstein participou, então, do evento de extensão intitulado Panoramas da toxicomania, promovido pelo Programa de Pós-Graduação em Educação da UFPR. Neste evento, a atividade intitulada Discutindo com o Dr. Claude Olievenstein foi coordenada pelo professor Bento. Após esta segunda visita de Olievenstein a Curitiba, um grupo de professores e profissionais técnicos da UFPR, sob a liderança do professor Bento, criou o CET - Centro de Estudos das Toxicomanias Dr. Claude Olievenstein -, em 19 de outubro de 1999, enquanto unidade diretamente vinculada ao Setor de Ciências Humanas, Letras e Artes da UFPR.

\section{A criação do CET da UFPR}

O CET foi fundado em 19/10/1999 por um grupo de professores e profissionais técnicos da UFPR, sob a liderança do Prof. Bento do Departamento de Psicologia, o qual foi Diretor deste centro desde a sua criação até 2/5/05.

Compreendia-se, por um lado, que as toxicomanias remetiam à ideia de uma paixão que fracassara numa evolução que as levariam ao encontro de um verdadeiro objeto de amor humano e, por outro lado, que tal destino 
patológico constituía uma perspectiva de todo ser humano. Explicava-se melhor esta ideia com as seguintes questões: Qual era o ser humano que nunca possuiu uma destas paixões "mortais" que se manifestavam, mais claramente, sobretudo nos momentos de frustração e de decepção quanto a alguma expectativa afetiva narcísica, sob a forma de um ódio "mortal" (reverso da paixão que não deixava de remeter ao mesmo significado de paixão, ainda que às avessas) do objeto, de um desejo de nunca mais vê-lo? Qual era o ser humano que nestas situações de perda de tal paixão, sem que esta fosse substituída pelo amor, pelo menos uma vez, não se viu as voltas com o desejo de afogar as mágoas numa bebedeira impessoal e anobjetal (no sentido do investimento não num objeto humano, mas sim na bebida), igualmente expressiva de um ódio "mortal", ainda que aqui já não se tratasse mais da hétero-agressividade evidente na questão anterior, mais sim de uma autoagressividade? Sendo assim, as toxicomanias eram vistas como uma doença narcísica, social por excelência, o que remetia a importância do tratamento desta problemática que a todos atingia, ou, pelo menos, podia atingir.

Portanto, na ótica teórica psicanalítica do CET, no período de outubro de 1999 a maio de 2005, fazia-se uma analogia entre as toxicomanias e as paixões. $\mathrm{O}$ que permitia tal aproximação era se entender existir uma função psíquica inconsciente semelhante nas duas: excesso do "narcisismo autoerótico", tanto nas toxicomanias, como nas demais paixões em geral - para o detalhamento das discussões teóricas a esse respeito, ver Bento (1993, 1994a, 1994b, 1995, 1996, 1998, 1999, 2006a, 2007a, 2008).

Para o leitor interessado em se aprofundar nas pesquisas sobre as toxicomanias, segundo uma ótica psicanalítica, realizadas por alunos do CET em parceria com seus professores orientadores, sugere-se ver principalmente: Mansilla e Bento (2006); Gomes, Guimarães e Bento (2007); Guimarães e Bento (2007); e Stacechen e Bento (2008).

As toxicomanias eram pesquisadas no CET principalmente através dos métodos da semiologia e do estudo de caso. Para os leitores interessados na teoria destes dois métodos de pesquisa, sugere-se ver principalmente: Bento (2006b, 2007b, 2007c) e Guimarães e Bento (2008).

4. Quem podia participar do CET da UFPR neste período de outubro de 1999 a maio de 2005?

Pelo Art. 3. ${ }^{\circ}$ do Regimento Interno do CET, era possível a participação de alunos, professores e profissionais da UFPR, de qualquer departamento, curso ou unidade, interessados na problemática das toxicomanias, abordada segundo qualquer enfoque teórico, em debate com a psicanálise. Algumas atividades do CET também eram abertas à comunidade externa, como, por exemplo, os Seminários Avançados do CET. Para participar, o interessado devia formalizar sua demanda junto a Direção do CET.

5. Como o CET da UFPR beneficiava a comunidade externa no período de outubro de 1999 a maio de 2005?

Ofertando tratamento e prevenção das toxicomanias, sem fins lucrativos. A clientela era atendida por acadêmicos da UFPR, sob a supervisão dos professores membros do CET. O CET também realizava palestras, cursos e seminários abertos à comunidade externa.

6. Quais linhas teóricas norteavam o funcionamento do CET da UFPR no período de outubro de 1999 a maio de 2005?

Além da Psicanálise, que era o enfoque teórico principal, o CET, enquanto centro inter e multidisciplinar, desenvolvia pesquisas e seminários dentro das seguintes linhas teóricas: Neuropsicologia, Sociologia Clínica, Psicologia Sistêmica e Psicologia Fenomenológica. O CET estava aberto a qualquer abordagem teórica das toxicomanias, na condição de que se pudesse estabelecer um debate com a psicanálise. 
7. A formação universitária em Psicanálise das Toxicomanias e Debate Inter e Multidisciplinar ofertada ao aluno da UFPR pelo CET no período de outubro de 1999 a maio de 2005

Anualmente o CET ofertava ao aluno da UFPR três anos ou níveis de uma formação na área da Psicanálise das Toxicomanias e Debate Inter e Multidisciplinar:

7.1 - $1 .^{\circ}$ ano - Nível Básico: Destinado aos alunos da UFPR interessados em realizar estágios introdutórios de pesquisa. Os alunos podiam se vincular ao CET através de disciplinas curriculares obrigatórias de cursos de graduação, de Estágio Voluntário (com cadastro na Prograd - Pró-Reitoria de Graduação), da Extensão Universitária (com cadastro na Proec - Pró-Reitoria de Extensão e Cultura), e/ou da Iniciação Científica (com cadastro na PRPPG - Pró-Reitoria de Pesquisa e Pós-Graduação).

7.2 - 2. ${ }^{\circ}$ ano - Nível Intermediário:

Destinado aos alunos da UFPR que pretendiam se aprofundar nas pesquisas iniciadas no nível básico. Em geral os discentes desse nível eram incentivados a transformar suas pesquisas introdutórias do nível anterior em monografia de conclusão de curso de graduação. Como no nível anterior, aqui o aluno também podia se vincular ao CET através de disciplinas curriculares obrigatórias de cursos de graduação, de Estágio Voluntário, da Extensão Universitária, e/ou da Iniciação Científica.

7.3 - 3..$^{\circ}$ ano - Nível Avançado: Voltado aos graduados, bacharéis em psicologia ou psicólogos, interessados no Curso de Especialização em Psicanálise das Toxicomanias ofertado pelo CET, o qual era essencialmente prático, pois valoriza estágios de pesquisa, de ensino e de extensão clínica. Este curso possuía 570 horas e funcionou anualmente, repetindose três vezes consecutivas, respectivamente nos anos letivos de 2002, 2003 e 2004.

8. Os Programas do CET da UFPR no período de outubro de 1999 a maio de 2005

Os Programas do CET ofertavam atividades voltadas para a formação dos alunos da UFPR, abrangendo os três níveis acima destacados. Eram eles:

8.1 - No Campo da Pesquisa Universitária: 1 - Diretório de Grupo Lattes, cadastrado no CNPq com o nome: Centro de Estudos das Toxicomanias Dr. Claude Olievenstein, desde abril de 2000; 2 - Programa de Pesquisas em Psicanálise das Toxicomanias e Debate Multidisciplinar do CET, cadastrado no Banco de Pesquisas da PRPPG da UFPR sob o número 2000007052, desde agosto de 1999.

8.2-NoCampodoEnsinoUniversitário: 1 - Programa de Seminários de Pesquisa do CET: semanais (Seminários Clínicos, para supervisão dos casos atendidos pelos alunos; Seminários Teórico-Clínicos, para a apresentação, discussão e orientação das pesquisas em andamento dos alunos, além de ser uma oportunidade para aprofundar questões teóricas sobre a temática da Pesquisa em Psicanálise das Toxicomanias e Debate Multidisciplinar); mensais (Seminários Avançados, para as defesas públicas das monografias de conclusão de curso de graduação e de especialização dos alunos, ou para a apresentação e discussão das pesquisas de profissionais especializados da comunidade externa, de diversas áreas, convidados para discutir temas da área das toxicomanias); bimestrais, semestrais e anuais (Seminários para avaliação dos alunos, dos professores e das atividades desenvolvidas no CET); 2 - Programa de Monitoria Voluntária de Disciplinas de Pesquisa: os discentes mais experientes (do 2. e 3. ${ }^{\circ}$ anos da formação do CET) atuavam como monitores voluntários de disciplinas curriculares de pesquisa, auxiliando assim os iniciantes (do 1. ${ }^{\circ}$ e 2. ${ }^{\circ}$ anos da formação do CET), metodologia esta que, além de introduzir os alunos no campo do magistério superior, contribuía para estimular o espírito de equipe e o ambiente de cooperação do CET; 3 - Programa de Cursos de Extensão e de Disciplinas Curriculares Obrigatórias ou Optativas, desenvolvidos no CET, no qual se abordava, de um ponto de 
vista teórico, temáticas como: Psicanálise das Toxicomanias; Psicopatologia Psicanalítica; Psicanálise do Adolescente; etc.; 4 - Programa de Estágios Curriculares (de pesquisa; de clínica; de ensino; etc.) desenvolvidos no CET; 5 - Programa de Eventos de Extensão, onde os alunos participavam apresentando e discutindo suas pesquisas desenvolvidas no CET; 6 - Programa de Bolsas ou Voluntariado: de Iniciação Científica; de Extensão; de Estágio (de pesquisa; de clínica; etc.); de Permanência; etc.; 7 - Programa de Pós-Graduação Lato-Sensu, que ofertava anualmente o Curso de Especialização em Psicanálise das Toxicomanias do CET.

8.3 - No Campo da Extensão Universitária: Programa de Extensão Universitária do Centro de Estudos das Toxicomanias Dr. Claude Olievenstein: Pesquisa, Ensino, Tratamento e Prevenção - cadastrado na PROEC da UFPR sob o número 037/00, desde abril de 2000. Realizaram-se neste Programa as seguintes atividades clínicas de extensão: psicoterapia de base analítica individual; psicoterapia analítica de grupo; grupos operativos voltados para a prevenção das toxicomanias com crianças, adolescentes e adultos; grupo de orientação a pais ou responsáveis de adolescentes atendidos; oficina de arte com adolescentes; ludoterapia em grupo com crianças; psicoterapia de famílias e de casais; etc.

9. Avaliação das ações desenvolvidas no CET da UFPR no período de outubro de 1999 a maio de 2005

O CET atingiu o objetivo de integrar Pesquisa, Ensino e Extensão, próprio das universidades em geral. Pois ofertou, neste período, de forma regular e articulada, atividades destas três áreas.

Quanto ao objetivo do Ensino, foram ofertadas pelo CET as seguintes atividades acadêmicas: cursos teóricos, orientações de pesquisa, supervisões em psicologia clínica, supervisões de monitores, três cursos de especialização em psicanálise das toxicomanias, etc. Muitos alunos da UFPR participaram simultaneamente de mais de uma das atividades.

Sobre o objetivo da Extensão Clínica para tratamento e prevenção das toxicomanias, foram atendidos diversos pacientes da comunidade externa a UFPR, com problemáticas de compulsões diversas: às drogas, ao álcool, ao relacionamento amoroso "tóxico", à comida, ao sexo, ao trabalho, ao consumo, etc. Esta clientela era atendida por alunos da UFPR, sob a supervisão de professores da mesma universidade. Para realizar este atendimento, essencialmente psicológico e de inspiração psicanalítica, foram utilizadas as seguintes técnicas: psicoterapia de base analítica individual de adultos; psicoterapia de base analítica de casal e de grupo de adolescentes; grupo de orientação aos pais dos adolescentes atendidos; $e$ oficina de arte-terapia para adolescentes.

Atingiu-se também, o objetivo de realizar pesquisas, que foram simultaneamente teóricas e clínicas, a partir da ótica principal da psicanálise das toxicomanias em debate multidisciplinar. Os resultados dessas pesquisas foram apresentados em diversos eventos científicos, tais como: nos Seminários de Pesquisa do CET da UFPR; nos EVINCIs - Eventos de Iniciação Científica promovidos pela PRPPG - Pró-Reitoria de Pesquisa e Pós-Graduação da UFPR; nos ENECs - Encontros de Extensão e Cultura promovidos pela Proec-Pró-Reitoria de Extensão e Cultura da UFPR, ou outros eventos científicos promovidos por diferentes instituições estaduais e nacionais de fora da UFPR. Aqui ocorria uma ação conjunta e integrada de professores e alunos do CET da UFPR.

Procurou-se, na organização dos seminários avançados do CET da UFPR, reunir alunos, professores e profissionais de linhas teóricas e especialidades diversas de dentro e de fora da UFPR, valorizando discussões conjuntas e integradas, com a finalidade de cultivar o espírito de equipe e o trabalho multi 
e interdisciplinar. Cabe destacar a participação aqui de diversos professores doutores e de renomados psicanalistas, lacanianos, ou ligados à IPA - International Psychoanalytical Association, alguns destes analistas didatas, nas bancas de exame de monografias de final de curso de graduação em psicologia e de curso de especialização em psicanálise das toxicomanias dos alunos do CET da UFPR. Deve-se dizer, que as monografias aqui em questão, eram realizadas e orientadas num período médio de três a quatro semestres letivos, o que contribuía para aprimorar a qualidade das pesquisas teórico-clínicas nelas relatadas.

Oreconhecimento, pelacomunidade, da excelência das pesquisas e práticas extensionistas do CET se fez notar em vários momentos. Destes, se pode especialmente destacar aquele quando, concorrendo a um concurso para ganhar um financiamento de pesquisa junto à Fundação Araucária, esta concedeu ao CET, entre 03/09/ 01 e 02/09/02, $\mathrm{R} \$$ 60.000,00 para seu projeto intitulado Programa de Pesquisas em Psicanálise das Toxicomanias e Debate Multidisciplinar. Além disso, o Programa de Extensão Universitária do

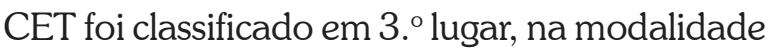
Painel, no 2. ${ }^{\circ}$ ENEC - Encontro de Extensão e Cultura - promovido pela PROEC da UFPR, em 13 e 14 de novembro de 2003. Finalmente, 14 pesquisas foram apresentadas por alunos do CET cadastrados no Grupo de Pesquisa Lattes deste

\section{REFERÊNCIAS}

BENTO, V. E. S. Tóxico, droga, toxicomania e adicção: uma introdução à etimologia segundo uma ótica psicanalítica. Jornal Brasileiro de Psiquiatria, Rio de Janeiro, v. 42, n. 7, p. 373-380, ago. 1993.

"Leonardo da Vinci e uma lembrança da sua infância": considerações sobre o narcisismo em Freud e sobre a paixão amorosa "tóxica" a partir de Freud. Temas: teoria e prática do psiquiatra, São Paulo, v.24, n. 47, p. 94-113, jan.-jun. 1994a.

Três ensaios sobre a teoria da sexualidade: considerações sobre o conceito de narcisismo em Freud (1905) e sobre a paixão amorosa "tóxica" a partir de
Centro, no $12 .^{\circ}$ EVINCI - Evento de Iniciação Científica promovido pela PRPPG da UFPR, em 13 e 14 de outubro de 2004, obtendo-se as seguintes classificações: um $1 .^{\circ}$ lugar, dois $2 .^{\text {os }}$ lugares e um $3 .^{\circ}$ lugar.

\section{Conclusão}

Este artigo relatou a experiência profissional do Prof. Dr. Victor Eduardo Silva Bento de criação e direção do CET - Centro de Estudos das Toxicomanias Dr. Claude Olievenstein: Pesquisa, Ensino, Tratamento e Prevenção, da UFPR, no período de 19/10/99 a 2/5/05. Narrouse a pré-história deste Centro e seu nascimento vinculado ao Setor de Ciências Humanas, Letras e Artes. Desde o início de sua história, o CET possuía um Acordo de Cooperação com o Centre Médical Marmottan / Paris, Centro este, na ocasião da criação do CET, sob a direção do Prof. Dr. Claude Olievenstein. Foram também descritas as atividades de Pesquisa, Ensino e Extensão Clínica do CET, segundo a orientação teórica principal da psicanálise, em debate multidisciplinar. Finalmente, avaliou-se as ações desenvolvidas no CET no mesmo período, destacando-se os prêmios e o financiamento recebidos pelo Centro.

Freud. Revista ABP-APAL, São Paulo, v.16, n. 4, p.154164, out.-dez. 1994b.

O presidente Schreber, um caso de paranóia: considerações sobre o narcisismo em Freud (1911) e sobre a paixão amorosa "tóxica" a partir de Freud. Informação Psiquiátrica, Rio de Janeiro, v.14, n.1, p.27-35, jan.-mar. 1995.

La passion amoureuse "toxique": une approche psychanalytique à partir de la sémiologie et du narcissisme chez Freud. 472 p. Thèse (Doctorat de Psychopathologie Fondamentale et Psychanalyse) - Université Paris VII, Paris, France, 1996. 
. O narcisismo em Freud e paixão "tóxica" a partir de Freud. Psicologia em Estudo, Maringá, Paraná, v. 3, n. 2, p.1-37, jul.-dez. 1998.

Pour introduire une "sémiologie psychanalytique" de la notion de "toxicomanie" dans l'approche médicale. Interventions: Revue de l'ANIT - Association Nationale des Intervenants en Toxicomanie, Saint-Genis-Laval, France, n. 68, p.16-22, mars 1999.

Tóxico e adicção comparados a paixão e toxicomania: Etimologia e Psicanálise. Psicologia USP, São Paulo, v.17, n.1, p.181-206, jan.-abr. 2006a.

Seria a semiologia de Saussure fundamento $e$ justificativa para o método de pesquisa de revisão de literatura em psicanálise? Estudos de Psicologia, Campinas, São Paulo, v. 23, n. 4, p. 407-423, out.-dez. 2006b.

Para uma semiologia psicanalítica das toxicomanias: adicções e paixões tóxicas no Freud pré-psicanalítico. Revista Mal-estar e Subjetividade, Fortaleza, v. 7, n. 1, p. 89-121, mar. 2007a.

. Totem e Tabu: uma "semiologia psicanalítica" em Freud? Estudos de Psicologia, Campinas, São Paulo, v. 24, n. 3, p. 397-406, jul.-set. 2007 b.

. Existiria uma "semiologia psicanalítica" em Lacan? Aletheia, Canoas, Rio Grande do Sul, n. 25, p.177190, jan.-jun. 2007c.

- Para uma semiologia psicanalítica da paixão na Antigüidade grega e seus sentidos adictivo e tóxico. Psicologia USP, São Paulo, v.19, n. 2, p.129-158, abr.jun. 2008.

GOMES, M. M.; GUIMARÃES, M. A. DE M.; BENTO, V. E. S. Da lei no Estatuto da Criança e do Adolescente a uma psicanálise do adolescente em conflito com a Lei.
Revista de Estudos Criminais, Rio Grande do Sul, v. 7. n. 24, p. 81-104, jan.-mar. 2007.

GROSSI, F. T. CMT: Centro Mineiro de Toxicomania. Relatório não publicado, Fundação Hospital do Estado de Minas Gerais/Secretaria Estadual de Saúde, Belo Horizonte, Minas Gerais, (199?).

. Centro Mineiro de Toxicomania: uma experiência singular. In. QUINET, A. (Org.). Psicanálise e psiquiatria: controvérsias e convergências. Rio de Janeiro: Rios Ambiciosos, 2001. p.165-170.

GUIMARÃES, M. A. DE M.; BENTO, V. E. S. Seria a religião uma saída para a toxicomania? Uma abordagem psicanalítica. Psychê: Revista de Psicanálise, São Marcos, São Paulo, v.11, n. 21, p.105-118, jul.-dez. 2007.

GUIMARÃES, R. M.; BENTO, V. E. S. O método do "estudo de caso" em psicanálise. Psico, Porto Alegre, Rio Grande do Sul, v. 39, n. 1, p. 91-99, jan.-mar. 2008.

MANSILLA, N. K. R.; BENTO, V. E. S. Drogadicção: tentativa de suicídio e/ou elaboração? Revista do Departamento de Psicologia - UFF, Niterói, Rio de Janeiro, v.18, n. 2, p.11-28, jul.-dez. 2006.

SILVEIRA FILHO, D. X. DA. Drogas: Uma compreensão psicodinâmica das farmacodependências. São Paulo: Casa do Psicólogo, 1995. 80 p.

; GORGULHO, M. Dependência: compreensão e assistência às toxicomanias (uma experiência do PROAD). São Paulo: Casa do Psicólogo, 1996. 258 p.

STACECHEN, L. F; BENTO, V. E. S. Consumo excessivo e adicção na pós-modernidade: uma interpretação psicanalítica. Fractal: Revista de Psicologia, Rio de Janeiro, v. 20, n. 2, p. 421-436, jul.-dez. 2008.

Texto recebido em 14 de janeiro de 2009. Texto aprovado em 27 de março de 2009. 